\title{
Cirurgia valvar e coronária simultânea
}

Pablo M. A. POMERANTZEFF* ${ }^{*}$ Miguel Antônio MORETTI*, Paulo Moniz de Aragão PORCIUNCULA*, Carlos Manoel de Almeida BRANDÃO*, José Antônio F. RAMIRES*, Noedir A. G. STOLF*, Adib D. JATENE*

\section{RBCCV 44205-250}

POMERANTZEFF, P. M. A.; MORETTI, M. A.; PORCIUNCULA, P. M. A.; BRANDÃO, C. M. A.; RAMIRES, J. A. F.; STOLF, N. A. G.; JATENE, A. D. - Cirurgia valvar e coronária simultânea. Rev. Bras. Cir. Cardiovasc., 9 (4): 213-219-xxx, 1994.

RESUMO: Este trabalho analisa 172 pacientes consecutivos operados de cirurgia valvar e coronária simultânea, no período compreendido entre julho de 1980 e junho de 1989 . O número de pacientes submetidos a revascularização do miocárdio e tratamento cirúrgico da valva aórtica (RAo) foi de 95 , sendo que 75 foram submetidos a tratamento cirúrgico da valva mitral e revascularização do miocárdio (RMi). As lesōes valvares mais freqũentes foram a estenose aórtica, 44 casos de insuficiência mitral em 40 casos. $O$ número de anastomoses por paciente foi de 1,87 em média para os pacientes RMi e de 1,56 nos pacientes RAo. A mortalidade hospitalar foi de $9,8 \%$ sendo que a mortalidade para os pacientes RAo foi de $7,3 \%$ e para os pacientes RMi foi de $12 \%$. Não houve diferença significativa $\left(x^{2}=04423\right)$ entre a mortalidade dos pacientes portadores de insuficiência mitral isquêmica, operados eletivamente ou em cárater de emergência, sendo a mortalidade de $20 \%$ nos eletivos e de $37,5 \%$ nos de emergência. Não houve relação entre mortalidade $\theta$ número de anastomoses por paciente. Houve tendência de melhores resultados com relação à mortalidade nos pacientes RMi com cirurgia conservadora $\left(x^{2}=1,6382\right)$. A curva actuarial de sobrevida mostra $82,6 \% \mathrm{em}$ 19 semestres para os pacientes $\mathrm{RMi}, 90,4 \%$ para os pacientes RAo e $86,3 \%$ para o estudo global de pacientes. Com uma evolução de 5172 meses/pacientes, $75 \%$ apresentam-se em classe funcionall (NYHA).

DESCRITORES: valvas cardiacas, cirurgia; miocárdio, revascularização, cirurgia.

\section{INTRODUÇÃO}

Muitos são os pacientes que necessitam de cirurgia valvular e coronária simultânea (CVCS), sendo que alguns têm indicação predominantemente valvar e outros indicação fuandamentalmente de causa coronária.

Os progressos na proteção miocárdica, tática operatória e cuidados no pós-operatório têm permitido bons resultados nestes pacientes, resultados estes que, evidentemente, dependem de vários fatores, salientando-se, entre estes, a indicação eletiva ou de emergência, a etiologia das lesões, a extensão da arteriosclerose coronária, a função ventricular esquerda e o status hemodinâmico na operação. Os casos mais graves são representados pelos pacientes operados em cárater de emergência, devido à insuficiência mitral isquêmica.

\section{CASUÍSTICA E MÉTODOS}

Foram estudados 172 pacientes consecutivos submetidos à CVCS, operados no período compreendido entre julho de 1980 à junho de 1989. A idade média dos pacientes estudados foi de 55,9 anos, sendo a idade máxima de 82 anos e mínima de 31 anos. Houve predominância do sexo masculino (128 pacientes).

Com relação aos diagnósticos mais freqüentes, 44 pacientes apresentavam insuficiência coronária (ICo) e estenose aórtica (EAO), 40 ICo e insuficiência mitral (IMi), 21 ICo e insuficiência aórtica (IAO), 17 ICo e estenose mitral (EMi), 14 ICo e dupla lesão aórtica (DLAo), 7 ICo e dupla lesão mitral (DLMi) e $5 \mathrm{ICo}, \mathrm{IAO}$ e aneurisma de aorta ascendente, sendo que $2 \%$ dos pacientes eram reoperação em prótese e $2 \%$ reoperação em coronária. Treze pacientes

Trabalho realizado no Instituto do Coração do Hospital das Clínicas da Faculdade de Medicina da Universidade de São Paulo.

Recebido para publicação em dezembro de 1994.

* Do Instituto do Coração do Hospital das Clínicas da Faculdade de Medicina da Universidade de São Paulo.

Endereço para correspondência: Pablo Pomerantzeff. Av. Dr. Eneas de Carvalho Aguiar, 44. Divisão Cirúrgica. CEP: 05403-000 São Paulo, SP, Brasil. 
POMERANTZEFF, P. M. A.; MORETTI, M. A.; PORCIUNCULA, P. M. A.; BRANDĀO, C. M. A.; RAMIRES, J: A. F.; STOLF, N. A. G.; JATENE, A. D. - Cirurgia valvar e coronária simultânea. Rev. Bras. Cir. Cardiovasc., 9 (4): 213-219, 1994.

apresentavam insuficiência mitral de causa isquêmica, sendo que 8 foram operados em caráter de emergência e 5 eletivamente.

Noventa e cinco pacientes foram submetidos a revascularização miocárdica e tratamento cirúrgico da valva aórtica (RAo), 75 a revascularização do miocárdio e tratamento cirúrgico da valva mitral (RMi), em 1 paciente foi realizada revascularização e dupla troca mitro-aórtica e, em outro, revascularização associada a troca mitral e retroca aórtica.

Em 82 pacientes submetidos a RAo foi feita substituição valvar e em 13 foi realizado procedimento conservador (Tabela 1).

Nos pacientes RMi foram realizadas 53 substituições valvares, 10 comissurotomias e 12 plásticas (Tabela 2). Na maioria dos pacientes em que foi realizada substituição valvar, foram utilizadas biopróteses, sendo empregada a de pericárdio bovino modelo InCor-EBM em 55 pacientes aórticos e 31 mitrais, e bioprótese porcina Biocor em 15 aórticos e 14 mitrais. Com relação aos pacientes mitrais, em 5 também tratamos a valva tricúspide, quatro vezes com plástica de De Vega e em $1 \mathrm{com}$ anel de Carpentier.

Nos pacientes submetidos à RMi com tratamento conservador da mitral foram realizados os seguintes procedimentos associados: 1 aneurismec- tomia de VE, 1 comissurotomia tricúspide, 2 retiradas de trombo de átrio esquerdo com fechamento de aurícula esquerda, 2 comissurotomias mitrais foram associadas à descalcificação da mesma, e, dos casos em que foi realizada plástica da mitral, 1 foi replastia.

O número de anastomoses por pacientes foi de 1,87 em média nos pacientes RMi e de 1,56 nos pacientes RAo, com um número mínimo de anastomoses de 1 e máximo de 5 , tanto nos RAo como nos RMi.

A torácica interna esquerda foi utilizada 12 vezes nas mitrais e 19 vezes nos aórticos e, em um paciente aórtico, foi utilizada dupla torácica interna.

Usando a classificação funcional da New York Heart Association (NYHA), $4(2,3 \%)$ pacientes estavam na classe funcional I, $17(9,8 \%)$ estavam na classe funcional II, $93(54 \%)$ classe funcional III e $58(33,7 \%)$ classe funcional IV.

A indicação da cirurgia foi predominantemente valvar em 78 pacientes $(45,3 \%)$, predominantemente de causa coronária em 43 pacientes $(25 \%)$ e de indicação tanto valvar quanto coronária em 51 $(29,6 \%)$ pacientes.

A mortalidade hospitalar foi definida como óbito nos primeiros 30 dias, ou na mesma internação.

TABELA 1

TRATAMENTO CIRÚRGICO DE VALVA AÓRTICA + REVASCULARIZAÇÃO DO MIOCÁRDIO

\begin{tabular}{|c|c|c|c|c|c|c|c|c|c|c|c|c|c|}
\hline \multicolumn{8}{|c|}{ PROCEDIMENTO VALVAR } & \multicolumn{6}{|c|}{ NÚMEROS DE ANASTOMOSES } \\
\hline \multirow{2}{*}{\multicolumn{7}{|c|}{ 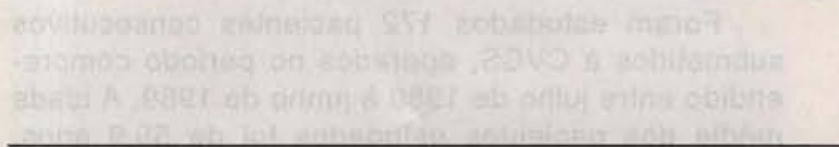 }} & est & \multicolumn{4}{|c|}{$\begin{array}{c}\text { NÚMERO DE } \\
\text { P.S. }\end{array}$} & \multirow[t]{2}{*}{$\begin{array}{c}\text { A.T.I. } \\
\text { E. }\end{array}$} & \multirow[t]{2}{*}{$\begin{array}{c}\text { A.T.I } \\
\text { D. }\end{array}$} \\
\hline & & & & & & & & 1 & 2 & 3 & 4 & & \\
\hline \multicolumn{7}{|c|}{ Desbastamento valvar } & & 1 & 1 & - & - & 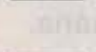 & 1 \\
\hline \multicolumn{7}{|c|}{ Plástica da valva aórtica } & & 1 & 1 & 1 & - & - & 1 \\
\hline \multicolumn{7}{|c|}{ Comissurotomia aórtica } & & 5 & 2 & 1 & - & 3 & - \\
\hline - Substituiçăo & $\begin{array}{l}\text { PB } \\
56\end{array}$ & $\begin{array}{c}\text { B } \\
17\end{array}$ & $\begin{array}{c}\mathrm{DM} \\
3\end{array}$ & $\begin{array}{c}\text { BS } \\
1\end{array}$ & $\begin{array}{c}\mathrm{SE} \\
2\end{array}$ & $\begin{array}{l}S \\
2\end{array}$ & $\underset{1}{O M}$ & 41 & 18 & 9 & 2 & 15 & - \\
\hline
\end{tabular}

P.S. - Pontes de Safena

A.T.I.E. - Artéria torácica interna esquerda

A.T.I.D. - Artéria torácica interna direita

PB - Pericárdio bovino (INCOR)

B - Válvula porcina (BIOCOR)

DM - Duramáter

BS - Björk-Shiley

SE - Starr-Edwards

S - Sorin

OM - Omnicarbon

* - Substituição + coronarioangioplastia intra-operatória (1 caso) 
POMERANTZEFF, P. M. A.; MORETTI, M. A.; PORCIUNCULA, P. M. A.; BRANDÃO, C. M. A.; RAMIRES, J. A. F.; STOLF, N. A. G.; JATENE, A. D. - Cirurgia valvar e coronária simultânea. Rev. Bras. Cir. Cardiovasc., 9 (4): 213-219, 1994.

TABELA 2

TRATAMENTO CIRÚRGICO DE VALVA MITRAL + REVASCULARIZAÇĀO DO MIOCÁRDIO

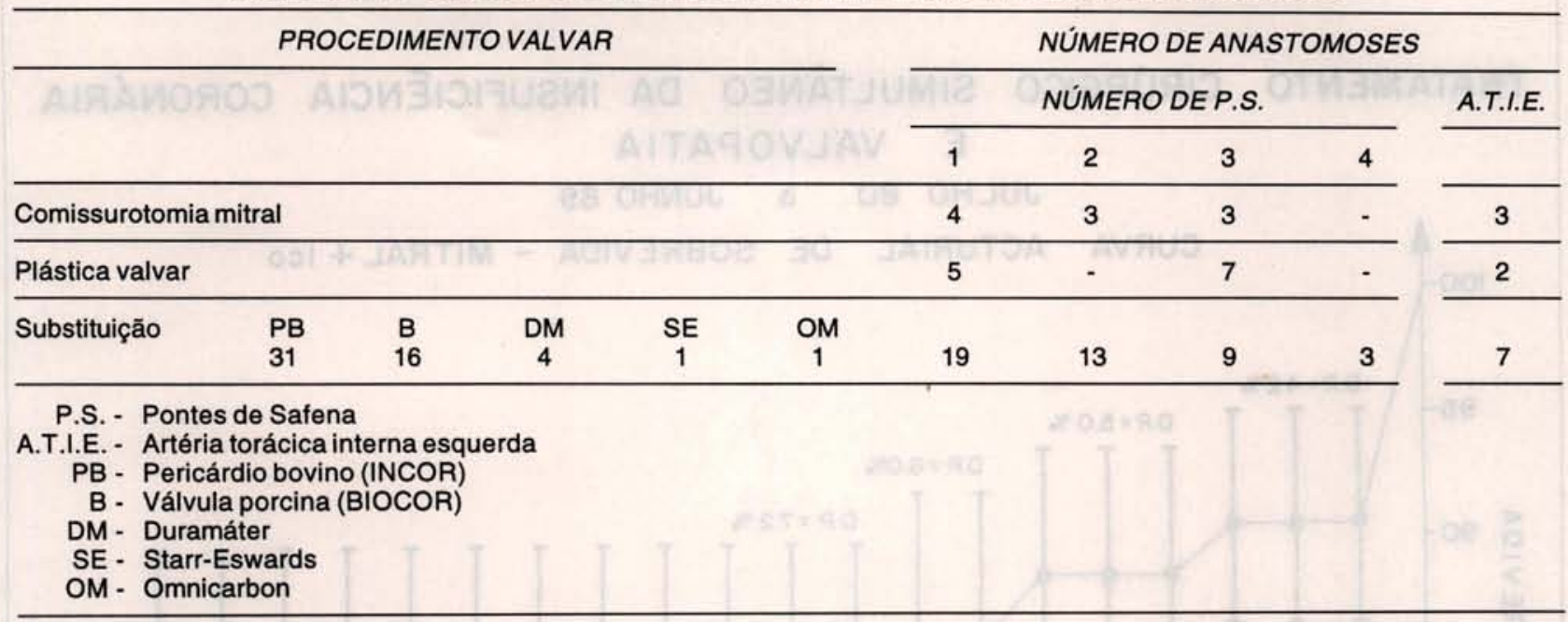

Com relação à proteção miocárdica, em todos os pacientes aórticos foi utilizada solução cardioplégica tipo St. Thomas, sendo que em alguns pacientes mitrais foi utilizado o pinçamento intermitente.

\section{RESULTADOS}

A mortalidade hospitalar global foi de $9,8 \%$ correspondendo a 17 pacientes em 172, a mortalidade nos pacientes RMi foi de $12 \%$, 9 pacientes em 75 , e a mortalidade dos pacientes RAo foi de $7,36 \%$, 7 pacientes em 95 . Um paciente mitral e 1 paciente aórtico que foram a óbito eram reoperações. Considerando-se os pacientes submetidos a cirurgia conservadora valvar, no global a mortalidade foi de $5,7 \%$ correspondendo a 2 pacientes, sendo 1 mitral e 1 aórtico. A mortalidade dos pacientes RMi em cirurgia conservadora foi de $4,5 \%, 1 \mathrm{em} \mathrm{22,} \mathrm{e} \mathrm{a}$ mortalidade dos pacientes RAo com cirurgia conservadora foi de $7,6 \%, 1$ em 13 .

Analisando os resultados de mortalidade na cirurgia de RMi com substituição valvar comparativamente aos de RMi com cirurgia conservadora através do Qui-Quadrado, encontramos uma tendência de melhores resultados com a cirurgia conservadora $\left(x^{2}=1,6382\right)$.

Considerando as insuficiências mitrais isquêmicas, nas cirurgias eletivas a mortalidade foi de $20 \%, 1$ paciente em 5 , e nas cirurgias realizadas em caráter de emergência foi de $37,5 \%$ correspondendo a 3 pacientes em 8 , resultado este estatisticamente não significativo $\left(x^{2}=04423\right)$.
Dos pacientes submetidos a dupla troca, 1 paciente submetido a retroca aórtica e troca mitral faleceu no pós-operatório imediato.

Nos pacientes que foram a óbito, o número de anastomoses por paciente foi de 1,8 nos pacientes RMi, 1,5 nos pacientes RAo em média, não havendo, portanto, relação do número de pontes com o óbito.

A causa mais freqũente de óbito no pós-operatório imediato foi o baixo débito com 2 casos associados a coma, houve 1 caso de edema agudo de pulmão não cardiogênico em paciente submetido à RAo com desbastamento da valva aórtica e ocorreu 1 infarto transmural hemorrágico em paciente RMi com substituição valvar.

Com relação às complicações mais freqũentes no pós-operatório imediato, 10 pacientes RMi apresentaram baixo débito transitório com utilização de balão intra-aórtico em 2 deles. Três pacientes RMi apresentaram acidente vascular cerebral transitório. Nos pacientes RAo, em 8 ocorreu baixo débito, sendo 1 por infarto agudo do miocárdio, e a utilização do balão intra-aórtico se limitou a 1 caso.

No estudo anatomopatológico das valvas retiradas, $65 \%$ dos pacientes aórticos apresentavam valvulite crônica calcificada, $10 \%$ degeneração mucóide, $5 \%$ valvulite crônica inespecífica e $5 \%$ valvulite degenerativa com depósito de amilóide. Nas mitrais, $37 \%$ apresentavam degeneração mucóide, $18 \%$ valvulite crônica inespecífica, $18 \%$ valvulite crônica fibrosada, $14 \%$ valvulite crônica calcificada e $9 \%$ valvulite crônica reumatismal. 


\section{TRATAMENTO CIRÚRGICO SIMULTÂNEO DA INSUFICIÊNCIA CORONÁRIA E VALVOPATIA JULHO 80 a JUNHO 89}

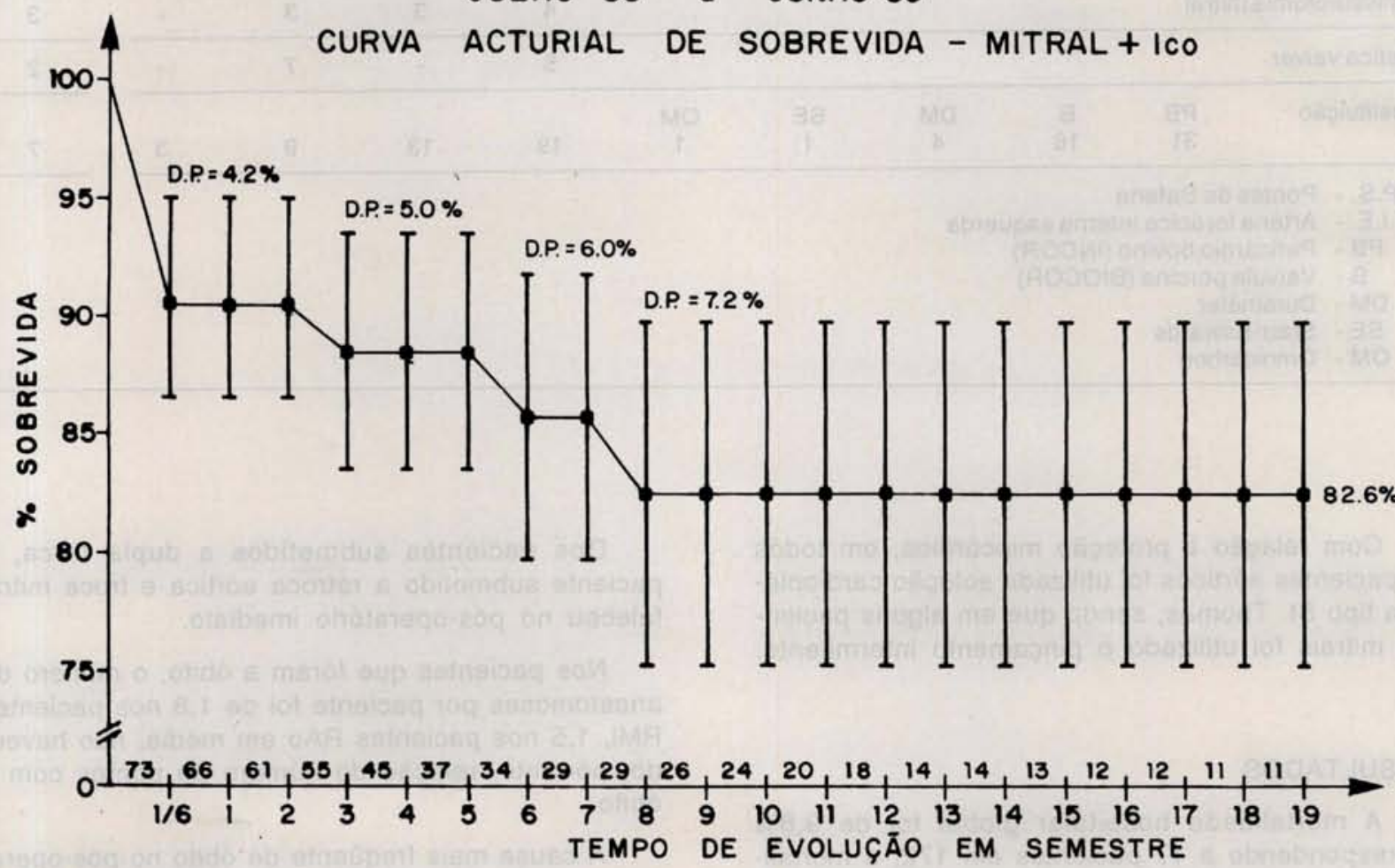

Foram acompanhados, no pós-operatório, 143 pacientes, correspondendo a $92 \%$ do total. Nos pacientes com próteses mecânicas, ocorreram 0,45 episódios de tromboembolismo por 100 pacientes/ ano. A retroca de próteses em pacientes portadores de próteses biológicas foi de 0,17 episódios por 100 pacientes/ano e de 1,3 episódios por 100 pacientes/ ano para as próteses mecânicas.

A análise da curva actuarial para pacientes RMi mostra $82,6 \%$ de sobrevida em 19 semestres (Gráfico 1), a sobrevida é de $90,4 \%$ para os pacientes RAo (Gráfico 2) e a sobrevida para o total de pacientes é de $86,3 \%$ (Gráfico 3 ).

Com um tempo de evolução de 5172 meses/ pacientes, 106 pacientes apresentaram-se com classe funcional I (NYHA), 32 em classe funcional II e 1 em classe funcional III, e no pós-operatório tardio ocorreram 4 óbitos (Gráfico 4).

\section{COMENTÁRIOS}

A mortalidade operatória dos pacientes submetidos a tratamento cirúrgico simultâneo da insuficiência coronária e valvopatia oscila, na literatura. Nos aórticos associados a revascularização LYTLE et alii ${ }^{7}$ relatam $5,9 \%$ de mortalidade hospitalar em uma série de 500 pacientes consecutivos e relatam 6 uma mortalidade de $7,3 \%$ nos pacientes mitrais associados a revascularização em 300 pacientes consecutivos.

TSAl et alii ${ }^{10}$, em uma série de pacientes septuagenários e octogenários operados simultâneamente para correção valvar e revascularização do miocárdio, relatam mortalidade global de $23 \%$, sendo a mortalidade para os septuagenários de $20 \%$ e para os octogenários de $38 \%$. Neste mesmo estudo, a mortalidade foi de $21 \%$ para os pacientes aórticos e de $50 \%$ para os pacientes mitrais. 
GRÁFICO 2

TRATAMENTO CIRÚRGICO SIMULTÃNEO DA INSUFICIÊNCIA CORONÁRIA E VALVOPATIA

JULHO 80 O JUNHO 89

CURVA ACTUARIAL DE SOBREVIDA - Aórtico + Ico

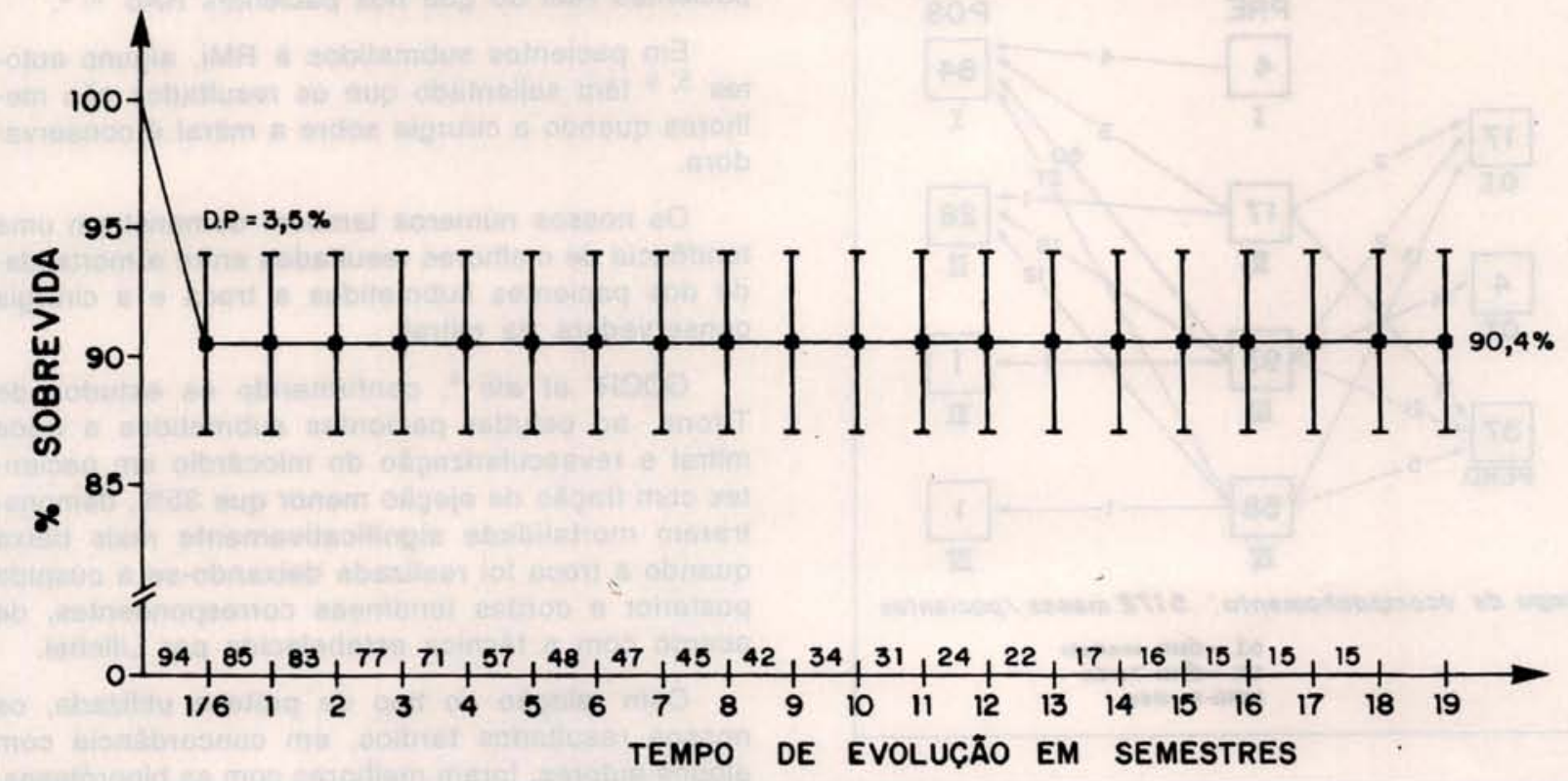

GRÁFICO 3

TRATAMENTO CIRÚRGICO SIMULTÂNEO DA INSUFICIÉNCIA CORONÁRIA E VALVOPATIA

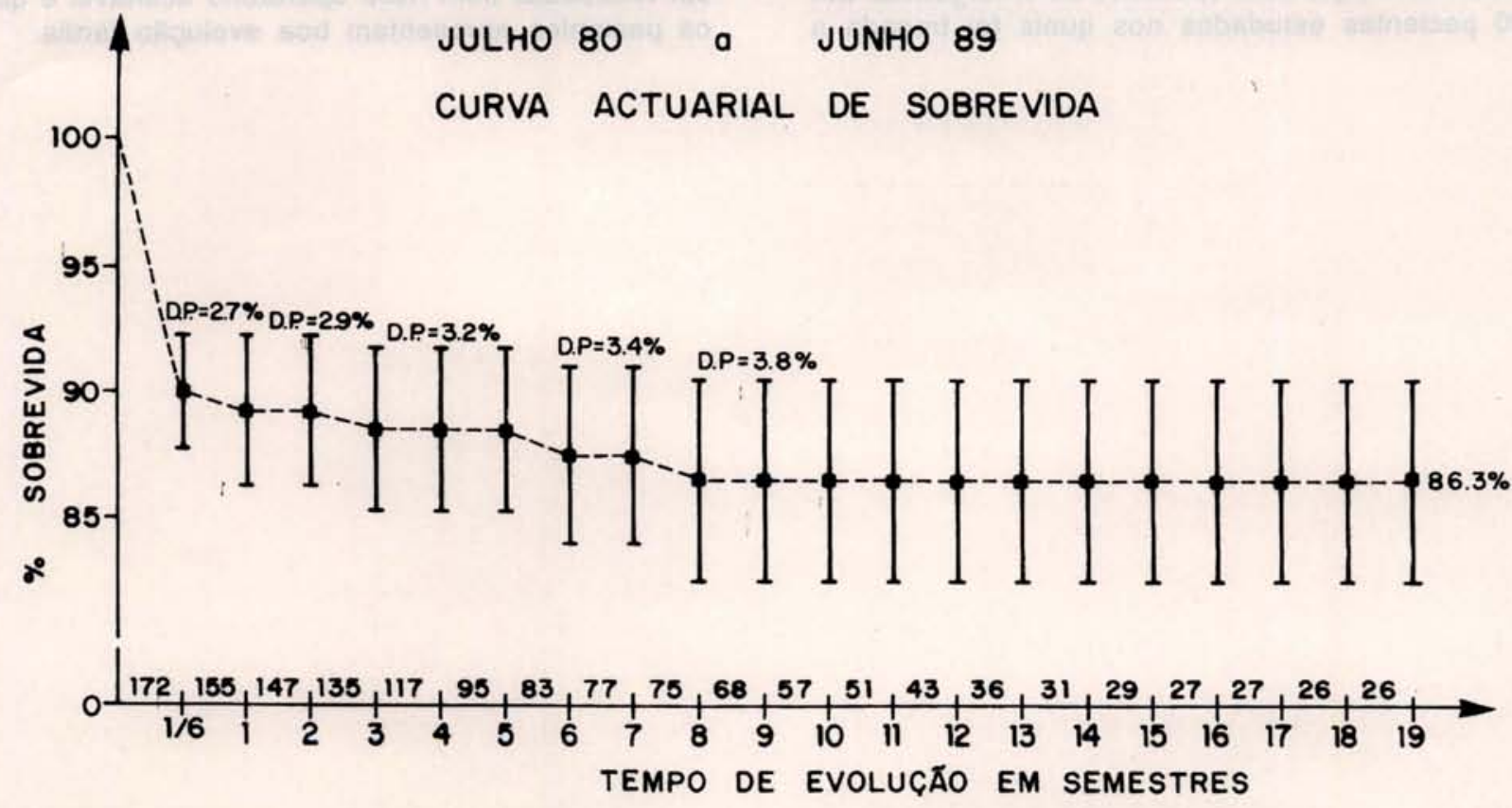


POMERANTZEFF, P. M. A.; MORETTI, M. A.; PORCIUNCULA, P. M. A.; BRANDĀO, C. M. A.; RAMIRES, J. A. F.; STOLF, N. A. G.; JATENE, A. D. - Cirurgia valvar e coronária simultânea. Rev. Bras. Cir. Cardiovasc., 9 (4): 213-219, 1994.

GRÁFICO 4

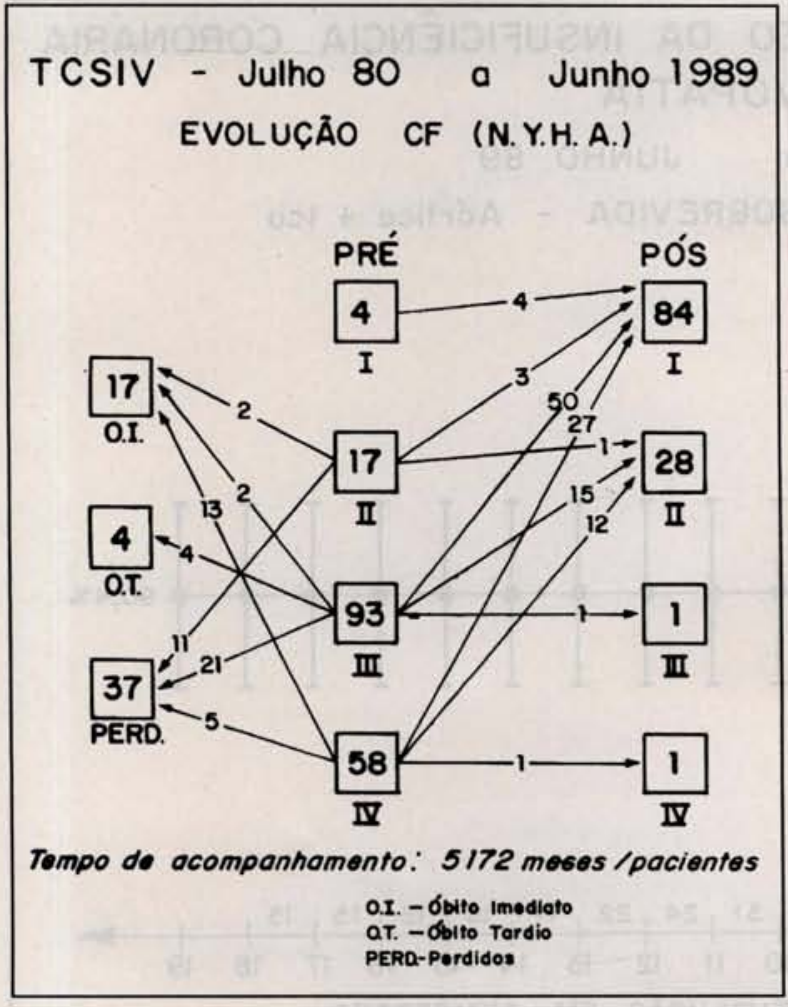

AKINS et alii ${ }^{1}$ apresentam mortalidade de $12 \%$ em uma série de 23 pacientes consecutivos submetidos a revascularização do miocárdio e dupla troca mitro-aórtica. DISESA et alii ${ }^{3}$ chamam a atenção para a alta mortalidade de pacientes com insuficiência mitral isquêmica operados de emergência. Em 100 pacientes estudados nos quais foi trocada a válvula mitral e realizada a revascularização do miocárdio, estes autores descrevem uma mortalidade global de $18 \%$, sendo que, nos casos operados de emergência, a mortalidade subiu para $60 \%$.

Os nossos resultados, em concordância com a literatura, mostram uma mortalidade maior nos pacientes RMi do que nos pacientes RAo ${ }^{2,8}$.

Em pacientes submetidos à RMi, alguns autores 5,9 têm salientado que os resultados são meIhores quando a cirurgia sobre a mitral é conservadora.

Os nossos números também demonstram uma tendência de melhores resultados entre a mortalidade dos pacientes submetidos a troca e a cirurgia conservadora da mitral.

GOOR et alii ${ }^{4}$, confirmando os estudos de Tirone, ao estudar pacientes submetidos a troca mitral e revascularização do miocárdio em pacientes com fração de ejeção menor que $35 \%$, demonstraram mortalidade significativamente mais baixa quando a troca foi realizada deixando-se a cúspide posterior e cordas tendíneas correspondentes, de acordo com a técnica estabelecida por Lillehei.

Com relação ao tipo de prótese utilizada, os nossos resultados tardios, em concordância com alguns autores, foram melhores com as biopróteses, salientando-se, porém, que, em nossa experiência, o número de próteses mecânicas foi muito pequeno.

Os nossos dados permitem concluir que a cirurgia de revascularização e valvar simultâneas podem ser realizadas com risco operatório aceitável e que os pacientes apresentam boa evolução tardia. 
POMERANTZEFF, P. M. A.; MORETTI, M. A.; PORCIUNCULA, P. M. A.; BRANDÃO, C. M. A.; RAMIRES, J. A. F.; STOLF, N. A. G.; JATENE, A. D. - Cirurgia valvar e coronária simultânea. Rev. Bras. Cir. Cardiovasc., 9 (4): 213-219, 1994.

\section{RBCCV 44205-250}

POMERANTZEFF, P. M. A.; MORETTI, M. A.; PORCIUNCULA, P. M. A.; BRANDÃO, C. M. A.; RAMIRES, J. A. F.; STOLF, N. A. G.; JATENE, A. D. - Combined coronary and valvular surgery. Rev. Bras. Cir. Cardiovasc., 9 (4): 213-219, 1994.

ABSTRACT: In the period between July 1980 and June 1989, 172 consecutive patients were submitted to combined coronary and valvular surgery. There were patients with predominantly valvular indications and others with predominant coronary indications. In 95 cases, myocardial revascularization and surgical treatment of the aortic valve (RAo) were simultaneously performed. Seventy five patients were submitted to myocardial revascularization and surgical treatment of the mitral valve (RMi). The more frequent valvular lesions were aortic stenosis ( 44 cases) and mitral insufficiency ( 40 cases). The mean number of anastomoses per patient was 1,87 for RMi cases and 1,56 for RAo patients. Overall hospital mortality was $9,8 \%$; RAo mortality was $7,3 \%$ and RMi mortality was $12 \%$. Mortality of patients with ischemic mitral insufficiency operated on electively $(20 \%)$ or in an emergency basis $(37,5 \%)$ was not statistically significant $\left(x^{2}=04423\right)$. There was not a relationship between mortality and the number of anastomoses per patient. $A$ tendency for better results regarding mortality was observed in RMi patients in which the valve was preserved $\left(x^{2}=1,6382\right)$. The survival actuarial curves in 19 semesters demonstrates a valve of $82,6 \%$ for RMi patients, $90,4 \%$ for RAo patients and $86,3 \%$ for the whole series. After evolution of 5172 months/patients, $75 \%$ were in functional class I (NYHA).

DESCRIPTORS: heart valves, surgery; myocardial revascularization, surgery.

\section{REFERÊNCIAS BIBLIOGRÁFICAS}

1 AKINS, C. W.; BUCKLEY, M. J.; DAGGETT, W. M.; AUSTEN, W. G.; HILGENBERG, A. D.; JACOBS, M. L. - Ten year follow-up of the Starr-Edwards prosthesis. Heart valve replacement: current status and future trends. In: RABAGO, G. \& COOLEY, D. A. (eds.) Futura Publishing Company Inc. Mount Kisko N. Y. 1987.

2 CZER, L. S. C. \& MATLOFF, J. M. - Combined valvular and coronary surgery. Chest, 90: 312-314, 1986.

3 DISESA, V. J.; COHN, L. H.; COLLINS, J. J.; KOSTER J. K.; VAN DEVANTES, S. - Determinants of operative survival following combined mitral valve replacement and coronary revascularization. Ann. Thorac. Surg. 34: 482-489, 1982.

4 GOOR, D. A.; MOHR, R.; LAVEE, J.; SERRAF, A.; SMOLINSKY, S. - Preservation of the posterior leaflet during michanical valve replacement for ischemic mitral refurgitation and complete myocardial revascularization. J. Thorac. Cardiovasc. Surg., 96: 253-260, 1988.

5 KAY, J. H.; ZUBIATE, P.; MENDEZ, M. A.; VANSTROM, N.; YOKOYAMA, T.; GHARAVI, M. A. - Surgical treatment of mitral insuficiency secondary to coronary artery disease. J. Thorac. Cardiovasc. Surg., 79: $12-18,1980$.

6 LYTLE, B. W.; COSGROVE, D. M.; GILL, C. C.; STEWART, R. N.; GOLDING, L. A. R.;
GOORMASTIC, M.; TAYLOR, P. C.; LOOP, F. D. Mitral valve replacement combined with myocardial revascularization: early and late results for 300 patients: $1970-1983$. Circulation., 71: 1179-1190, 1985.

7 LYTLE, B. W.; COSGROVE, D. M.; LOOP, F. D.; TAYLOR, P. C.; GILL, C. C.; GOLDING, L. A. R.; GOORMASTIC, M.; GROVES, L. K. - Replacement of aortic valve combined with myocardial revascularization: determinants of early and late risk for 500 patients: 1967-1981. Circulation., 68: 1149$1162,1983$.

8 MULLANY, C. J.; ELVEBACK, L. R.; FRYE, R. L.; PLUTH, J. R.; EDWARDS, W. D.; ORSZULAK, T. A.; NASSEF, L. A.; RINER, R. E.; DANIELSON, G. K. - Coronay artery disease and its management: influence on survival in patients undergoing aortic valve replacement. J. A. C. C., 10: 66-72, 1987.

9 ORSZULAK, T. A.; SCHAFF, H. V.; DANIELSON, G. K.; PIEHLER, J. M.; PLUTH, J. R.; FRYE, R. L.; MCGOON, D. C. - Mitral regurgitation due to ruptured chordae tendinae: early and late results of valve repair. J. Thorac. Cardiovasc. Surg., 89: 491-498, 1985.

10 TSAI, T. P.; MATLOFF, J. M.; CHAUX, A.; KASS, R. M.; LEE, M. E.; CZER, L. S. C.; DeROBERTIS, M. A.; GRAY, R. J. - Combined valve and coronary artery by pass procedures in septuagenarians and octogenarians: results in 120 patients. Ann. Thorac. Surg., 42: 681-684, 1986. 